\title{
Fair and QoS-oriented resource management in heterogeneous networks
}

\author{
Mustafa Cenk Ertürk ${ }^{1,2^{*}}$, Ismail Güvenç ${ }^{3}$, Sayandev Mukherjee ${ }^{2}$ and Hüseyin Arslan²
}

\begin{abstract}
In this paper, a heterogeneous network composed of femtocells deployed within a macrocell network is considered, and a quality-of-service (QoS)-oriented fairness metric which captures important characteristics of tiered network architectures is proposed. Using homogeneous Poisson processes, the sum capacities in such networks are expressed in closed form for co-channel, dedicated channel, and hybrid resource allocation methods. Then a resource splitting strategy that simultaneously considers capacity maximization, fairness constraints, and QoS constraints is proposed. Detailed computer simulations utilizing 3GPP simulation assumptions show that a hybrid allocation strategy with a well-designed resource split ratio enjoys the best cell-edge user performance, with minimal degradation in the sum throughput of macrocell users when compared with that of co-channel operation.
\end{abstract}

\section{Introduction}

The demand for wireless broadband data has been growing dramatically over the recent years, which introduces an important challenge for next-generation radio access networks. Recent predictions show that due to the increasing range of throughput-demanding applications on mobile devices, a global data growth as large as 33 times is forecasted by some analysts over the next 5 years, a large portion (70\% to $90 \%)$ of which will be mostly initiated from low-mobility users [1]. Moreover, the data consumed by applications of mobile devices such as smartphone and Google glasses will provide extreme burdens to cellular network providers since users will demand high data rates for their devices at anytime and anywhere. Although overlay of Wi-Fi networks in unlicensed spectrum complements cellular networks at residences, in office buildings, or at public hotspots in some use cases, it cannot provide a reliable and quality-ofservice (QoS)-oriented access for users [2]. On the other hand, since the traditional macrocellular networks are not built for these use cases, it will be extremely challenging to meet such demand for high data rates in the upcoming years.

*Correspondence: merturk@mail.usf.edu

1 Department of Electrical Engineering, University of South Florida, Tampa, FL 33620, USA

2 DOCOMO Innovations Inc., 3240 Hillview Avenue, Palo Alto, CA 94304, USA Full list of author information is available at the end of the article
In order to address these challenges, there has been an increasing interest to deploy low-power nodes within the coverage areas of macrocellular networks, such as picocells, femtocells, relay nodes, and distributed antenna systems. These networks, which are commonly referred as heterogeneous networks [3], can efficiently reuse the wireless resources (power, spectrum, hardware, available nodes and networks, etc.) due to low-power operation and, at the same time, maintain good link qualities with the end users due to the relatively shorter communication distances ${ }^{\mathrm{a}}$.

In heterogeneous networks, frequency resources can be allocated to different tiers in a co-channel (shared spectrum) or dedicated channel (split spectrum) ${ }^{\mathrm{b}}$ manner, or through a hybrid technique which is a combination of the two approaches. In the co-channel approach shown in Figure 1a, while the spectrum resources are fully reused in different tiers, cross-tier interference may cause crucial setbacks to the system. For example, macrocell users in the vicinity of closed subscriber group (CSG) femtocells are not allowed to connect to the femtocells, even if their link quality is good with these femtocells. Therefore, such macrocell users receive strong downlink interference from CSG femtocells and may fall into outage.

The split spectrum approach shown in Figure 1b, on the other hand, partitions the allocated spectrum between multiple tiers. Each tier can use its own segment of resource and therefore there is no cross-tier interference [4]. However, the amount of bandwidth available

\section{是 Springer}

(c) 2013 Ertürk et al: licensee Springer. This is an Open Access article distributed under the terms of the Creative Commons

Attribution License (http://creativecommons.org/licenses/by/2.0), which permits unrestricted use, distribution, and reproduction in any medium, provided the original work is properly cited. 


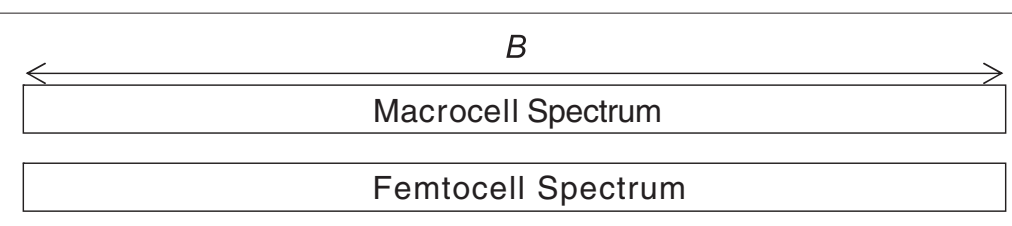

(a)

\begin{tabular}{|c|c|}
\hline$(1-\rho) B$ & $\rho B$ \\
\hline Femtocell Spectum & Macrocell Spectrum \\
\hline
\end{tabular}

(b)

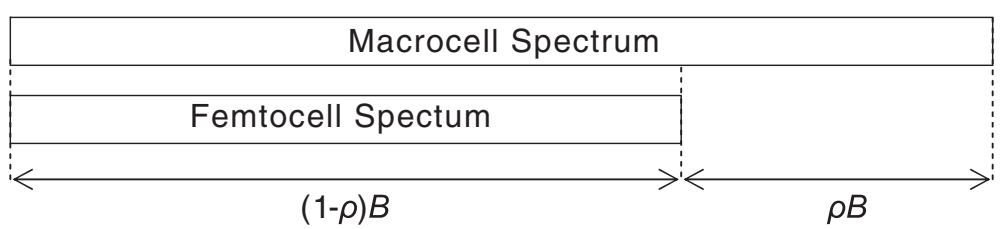

(c)

Figure 1 Resource allocation approaches in heterogeneous networks. (a) Co-channel, (b) dedicated channel, and (c) hybrid approach. Spectrum splitting ratio (SSR) is denoted by $\rho$. B indicates bandwidth.

to each tier is reduced. Hybrid methods as shown in Figure 1c use a mixture of co-channel and dedicated channel methods and aim to reuse the spectrum resources whenever feasible. For example, in [5] the macrocell users are dedicated to a component carrier $(\mathrm{CC})$, referred as the 'escape carrier', which is not used by the femtocell network. Any macrocell mobile station (mMS) which is close to a femtocell is scheduled within this escape carrier, if the interference observed from the femtocell network is above threshold. Hence, user outages are prevented by scheduling victim users in dedicated resources, while the spectrum is still reused in co-channel CCs. The resources within a certain $\mathrm{CC}$ may also be partitioned into smaller chunks for similar interference mitigation purposes [6].

Performance of dedicated channel and co-channel femtocell/macrocell networks have been investigated and compared through computer simulations in $[7,8]$. Both papers show that co-channel deployment increases the total system throughput at the expense of some degradation in the throughput of macrocell users that are close to the femtocells. However, impact of different spectrum splitting ratios (SSRs) on the overall network has not been studied in these works. Capacity cumulative distribution functions (CDFs) of indoor and outdoor users for different SSRs have been compared through computer simulations in [9], which shows that for certain scenarios, the performance close to the co-channel deployment can be obtained by appropriately setting the SSR value in a dedicated channel setting. Bharucha et al. investigate the impact of dynamic resource partitioning for downlink femto-to-macrocell interference avoidance for co-channel femtocell deployments in [6]. The simulation results show that co-channel deployment with dynamic resource partitioning can benefit from the frequency reuse property to achieve high throughputs, and femtocells can switch to orthogonal resource utilization when a close-by macrocell user is detected. However, so called X2 interface between the macrocell base station (mBS) and the femtocell base station (fBS) is assumed to be available in order to exchange the interference coordination information. In [10], an autonomous power control algorithm is introduced to enhance interference management in heterogeneous networks, and user equipments or UEs are proposed to be employed in the interference cancellation of broadcast interfering signals. As opposed to CSG access method, a shared access method which allows femtocells to allocate an adjustable number of time slots between home and cellular users is proposed in [11]. A survey on interference and resource management in femtocell networks can be found in [12].

One of the key aspects of spectrum allocation in heterogeneous networks is to define a metric to measure and evaluate the degree of fairness and QoS in the overall system [13]. The fundamental work in the area was done by Jain and Hawe [14], which analyzes all the properties of the fairness metric. Bandwidth assignment and scheduling-related optimization problems using fairness criteria were investigated in [15]. Utility-based fairness indices [16] have been widely recognized due to their flexibility for various application types. All of the above metrics are designed by considering a 
one-tier-coordinated deployed network. However, when the structure of the network is tiered, we propose that the metric should also include the heterogeneity of the network.

The goal of this paper is to provide a fairness metric for heterogeneous network architectures and to optimize the $\operatorname{SSR}(\rho)$ in dedicated channel and hybrid channel approaches, as in Figure 1b,c, considering the fairness and QoS constraints. First, the sum capacities of different tiers in a heterogeneous network are expressed in closed form for all approaches, and the capacity-maximizing spectrum splitting is investigated. To fairly allocate the resources to different tiers, a modified QoS-oriented fairness metric is introduced. This metric captures important characteristics of tiered network architectures such as the number of networks in each tier, the number of users in each network, and the QoS requirements of different tiers. Then a spectrum splitting strategy that simultaneously considers capacity maximization, fairness constraints, and QoS constraints is proposed. For different SSR values, sum capacities of macrocells and femtocells are obtained through analytical derivations and computer simulations and compared through various scenarios.

We can summarize the contribution of this paper as follows. (1) A modified fairness metric is introduced which can be used for resource management in tiered network structures such as heterogeneous networks. This metric is investigated in an example scenario, its properties are presented, and it is compared with the Jain's fairness metric used for homogeneous networks. (2) The sum capacity of hybrid channel approach resource management is analytically derived as well as the traditional methods such as co-channel and dedicated channel as a toolbox for comparisons. Simulation results are obtained to confirm analytical findings. (3) For the hybrid channel approach, a method for scheduling of users in macrocell is proposed utilizing max-min fair scheduling approach.

The remaining of this paper is organized as follows. In Section 2, the system model to provide total capacity of a macrocell-femtocell network is provided, and QoS-oriented fairness metric for tiered network structures is proposed. In Section 3, capacities of co-channel, dedicated channel, and hybrid channel approaches are derived using homogeneous Poisson processes (HPPs) and a max-min fair scheduler is introduced for hybrid channel approach. Numerical results for various scenarios are presented in Section 4, followed by concluding remarks in Section 5.

\section{Fairness metric and system model for heterogeneous networks}

Consider a two-tier macrocell-femtocell scenario (i.e., $T=2$ ), where macrocell-tier network is the tier-1 network and femtocell-tier network is the tier-2 network. We evaluate the total capacity of both tiers where femtocells are assumed to be randomly distributed within the coverage area of the given macrocell, and our goal is to maximize

$$
C_{\mathrm{Tot}}=\sum_{i=1}^{T} \sum_{j=1}^{N_{\mathrm{N}, i}} \sum_{k=1}^{N_{\mathrm{U}, i, j}} C_{i, j, k}
$$

while considering fairness metric and QoS parameter. Note that $N_{\mathrm{N}, i}, N_{\mathrm{U}, i, j}$, and $C_{i, j, k}$ are number of cells in $i$ th tier, number of users in the $i$ th tier and $j$ th cell, and rate of the $k$ th user in the $i$ th tier and $j$ th cell, respectively.

\section{Capacity of macrocell and femtocell}

The total capacity for the femtocell-tier (tier-2) network can be expressed as

$$
C_{\mathrm{Fem}}=C_{2}=\sum_{j=1}^{N_{\mathrm{N}, 2}} \sum_{k=1}^{N_{\mathrm{U}, 2, j}} \underbrace{B_{2, j, k} \log _{2}\left(1+\frac{P_{2, j, k}}{I_{2, j, k}+B_{2, j, k} N_{0}}\right)}_{C_{2, j, k}},
$$

where $B_{2, j, k}, P_{2, j, k}$, and $I_{2, j, k}$ denote bandwidth, received power, and interference power observed by the $k$ th user with the $j$ th femtocell, respectively; $N_{0}$ is the spectral density of noise; and $C_{2, j, k}$ is the capacity of femtocell user $k$ with the $j$ th femtocell.

Similarly, the total capacity of macrocell tier can be written as

$$
C_{\mathrm{Mac}}=C_{1}=\sum_{j=1}^{N_{\mathrm{N}, 1}} \sum_{k=1}^{N_{\mathrm{U}, 1, j}} \underbrace{B_{1, j, k} \log _{2}\left(1+\frac{P_{1, j, k}}{I_{1, j, k}+B_{1, j, k} N_{0}}\right)}_{C_{1, j, k}},
$$

where $B_{1, j, k}, P_{1, j, k}, I_{1, j, k}$, and $C_{1, j, k}$ denote bandwidth, received power, interference power, and capacity for the $k$ th macrocell user in the $j$ th macrocell, respectively. Both macrocell users (mMS) and femtocell users (fMS) are assumed to be distributed within each circular macrocell and femtocell area.

\subsection{QoS orientation and fairness metric for tiered networks}

In this section, we first define a fairness index and propose that a fair spectrum allocation can be achieved by considering the heterogeneous architecture of tiered networks. Then a QoS parameter is also added in the fairness metric to provide QoS orientation for the spectrum allocation. 


\subsubsection{Fairness index}

Jain's fairness index (JFI) [14] has been a widely used fairness criterion in the literature for resource allocation and can be written as

$$
f(x)=\frac{\left(\sum_{i=1}^{N} x_{i}\right)^{2}}{N \sum_{i=1}^{N} x_{i}^{2}},
$$

where $N$ denotes the total number of users, and $x_{i}$ denotes the received allocation for the $i$ th user. Some of the important properties of Equation 4 are as follows: (1) population size independence, (2) scale and metric independence, (3) boundedness $(f(x) \in[1 / N, 1], \forall x),(4)$ direct relationship, and (5) continuity (non-discrete).

Tiered network structures, such as those that include femtocells, picocells, and relay networks overlaid with a macrocell network, introduce a multi-dimensional resource allocation problem. In tiered networks, where users are distributed among tiers and the cells within each tier, providing a global fairness index for the entire system requires a modified fairness criteria. Consider a $T$-tiered architecture where each tier has several cells, similar to the one defined in Section 2.1 with the same notation. We propose that a tiered fairness index (TFI) in such a system should be as follows

$$
f_{\mathrm{TFI}}(\mathbf{C})=\frac{\left(\sum_{i=1}^{T} \sum_{j=1}^{N_{\mathrm{N}, i}} \sum_{k=1}^{N_{\mathrm{U}, i, j}} N_{\mathrm{U}, i, j} C_{i, j, k}\right)^{2}}{N_{\mathrm{Tot}} \sum_{i=1}^{T} \sum_{j=1}^{N_{\mathrm{N}, i}} \sum_{k=1}^{N_{\mathrm{U}, i, j}} N_{\mathrm{U}, i, j}^{2} C_{i, j, k}^{2}},
$$

where $\mathbf{C}$ denotes the set of capacities of all the users in all tiers, and $N_{\text {Tot }}$ is the total number of users in the entire system:

$$
N_{\mathrm{Tot}}=\sum_{i=1}^{T} \sum_{j=1}^{N_{\mathrm{N}, i}} N_{\mathrm{U}, i, j}
$$

The difference of (5) from the JFI is that it is a global fairness index for a tiered network and it provides a single fairness index by weighting the tiers and cells according to their number of users ${ }^{\mathrm{C}}$.

Using the notation, Equation 4 may be rewritten by changing $N$ with $N_{\text {Tot }}$ as follows:

$$
f_{\mathrm{JFI}}(\mathbf{C})=\frac{\left(\sum_{i=1}^{T} \sum_{j=1}^{N_{\mathrm{N}, i}} \sum_{k=1}^{N_{\mathrm{U}, i, j}} C_{i, j, k}\right)^{2}}{N_{\text {Tot }} \sum_{i=1}^{T} \sum_{j=1}^{N_{\mathrm{N}, i}} \sum_{k=1}^{N_{\mathrm{U}, i, j}} C_{i, j, k}^{2}} .
$$

Since the JFI in (7) does not consider the number of users in each cell for tiered scenarios, it does not satisfy the boundedness property (see Table 1 ). In other words,

\begin{tabular}{|c|c|c|}
\hline FI & Lower bound & Upper bound \\
\hline$f_{\mathrm{JF}}(\mathbf{C})$ & $1 / N_{\text {tot }}$ & No explicit solution ${ }^{a}$ \\
\hline$f_{\mathrm{TFI}}(\mathbf{C})$ & $1 / N_{\text {tot }}$ & 1 \\
\hline$f_{\mathrm{WJFI}}(\mathbf{C})$ & $\frac{\sum_{i=1}^{T} \sum_{j=1}^{N_{N, i}} 1 / N_{U, i, j}}{\sum_{i=1}^{T} N_{N, i}}$ & 1 \\
\hline$f_{\mathrm{QTFI}}(\mathbf{C})$ & $1 / N_{\text {tot }}$ & 1 \\
\hline
\end{tabular}
$f_{\mathrm{JFI}}(\mathbf{C})$ is no longer tightly bounded within $\left[1 / N_{\mathrm{Tot}}, 1\right]$. While the number of users in each cell varies, the
Table 1 Bounds for fairness indices

upper bound of JFI changes. This property will be discussed in an example case study later in Section 4.1 (see Figure 2).

Finding the JFI for each cell in each tier and obtaining its weighted summation could be another approach for a modified fairness index for tiered networks which satisfies the upper bound of 1 as opposed to JFI (see Table 1). The weighted sum JFI (WJFI) could be written as

$$
f_{\mathrm{WJFI}}(\mathbf{C})=\frac{1}{\sum_{i=1}^{T} N_{\mathrm{N}, i}} \times \sum_{i=1}^{T} \sum_{j=1}^{N_{\mathrm{N}, i}} \frac{\left(\sum_{k=1}^{N_{\mathrm{U}, i, j}} C_{i, j, k}\right)^{2}}{N_{\mathrm{U}, i, j} \sum_{k=1}^{N_{\mathrm{U}, i, j}} C_{i, j, k}^{2}} .
$$

However, WJFI does not consider the number of users in each cell and weighs the fairness with the total number of cells in the system $\left(\sum_{i=1}^{T} N_{\mathrm{N}, i}\right)$. For instance, if one cell (i.e., macrocell or each one of the femtocells) has twice the number of users compared to another cell, (8) does not consider this and provides equal weights for each cell. While this metric has an upper bound of 1 , it does not have a lower bound of $1 / N_{\text {Tot }}$. Table 1 shows that the lower bound of the equation is increasing with the decrease in the number of users in each cell, which is a very common case for a femtocell scenario.

\subsubsection{QoS-oriented TFI}

In tiered networks, it is typically expected that the tiers will have different QoS requirements. For example, in a macrocell-femtocell two-tier network, femtocell users are expected to have significantly better throughput compared to macrocell users due to better link qualities and larger spectrum resources. Therefore, the QoS characteristics of each tier should also be considered within the fairness index in order to have a better representation of fairness within the whole system. Let $\beta_{i}(i=2, \ldots, T)$ denote the QoS parameter defined as the ratio of the sum capacity in the first tier (e.g., macrocell tier) to the sum capacity in a different tier (e.g., femtocell tier)

$$
\beta_{i}=\frac{1 / N_{\mathrm{N}, 1} \sum_{j=1}^{N_{\mathrm{N}, 1}} \sum_{k=1}^{N_{\mathrm{U}, 1, j}} C_{1, j, k}}{1 / N_{\mathrm{N}, i} \sum_{j=1}^{N_{\mathrm{N}, i}} \sum_{k=1}^{N_{\mathrm{U}, i, j}} C_{i, j, k}}
$$




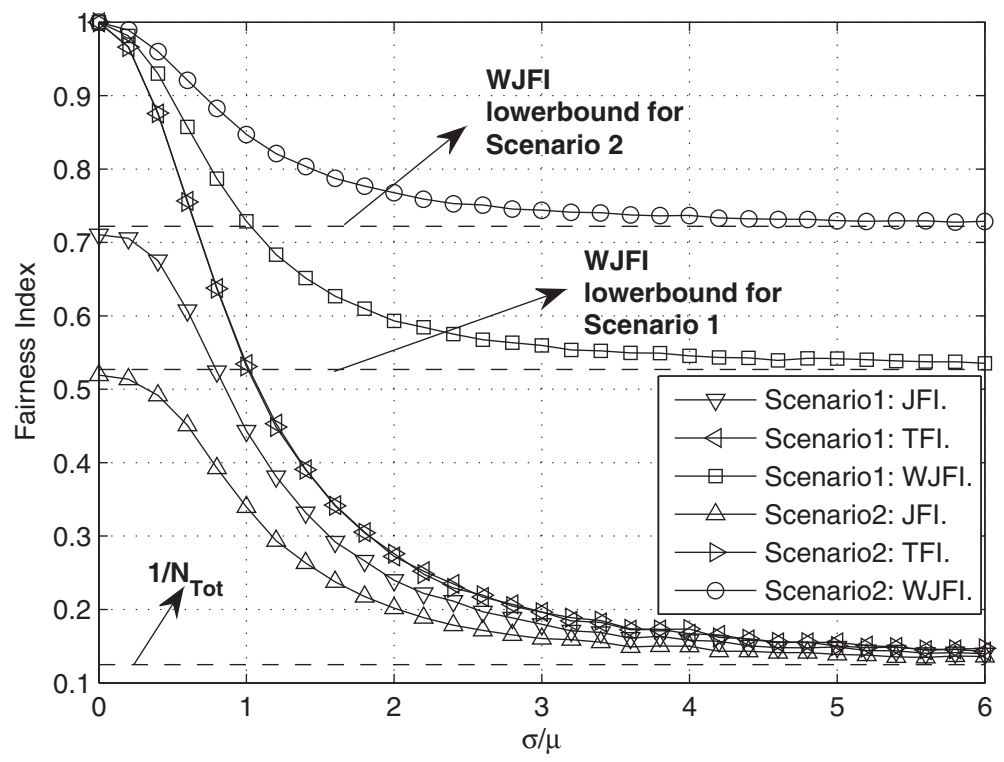

Figure 2 Fairness index vs. standard deviation $(\sigma) /$ mean $(\mu)$ for normally distributed resource allocations for each user.

where $\beta_{1}=1$. Using this QoS parameter, a modified version of the proposed fairness index in (5) can be written as

$$
f_{\mathrm{QTFI}}(\mathbf{C})=\frac{\left(\sum_{i=1}^{T} \sum_{j=1}^{N_{\mathrm{N}, i}} \sum_{k=1}^{N_{\mathrm{U}, i, j}} \beta_{i} N_{\mathrm{U}, i, j} C_{i, j, k}\right)^{2}}{N_{\mathrm{Tot}} \sum_{i=1}^{T} \sum_{j=1}^{N_{\mathrm{N}, i}} \sum_{k=1}^{N_{\mathrm{U}, i, j}} \beta_{i}^{2} N_{\mathrm{U}, i, j}^{2} C_{i, j, k}^{2}} .
$$

Note that (10) converges to (5) while $\beta_{i} \rightarrow 1(i=$ $2, \ldots, T)$. Moreover, if macrocell is the only tier in the system (i.e., $T=1, N_{\mathrm{N}, 1}=1$ ), then (5) converges to the Jain's fairness index given in (7). This proves that the provided equations are the modified versions of the JFI in order to satisfy the boundedness property within $\left[1 / N_{\text {Tot }}, 1\right]$. Table 1 summarizes the lower and upper bounds of the above-mentioned fairness indices.

It is important to note that the proposed fairness indices $\left(f_{\mathrm{TFI}}(\mathbf{C}), f_{\mathrm{QTFI}}(\mathbf{C})\right)$ are bounded and independent of the number of cells in the tiered network structure. JFI upper bound is not independent from the allocated resources; therefore, a closed-form expression could not be achieved. On the other hand, the lower bound of the WJFI depends on the number of cells and the number of users within the cells in each tier. Therefore, proposed fairness indices would provide a controlled metric for resource allocation problems in heterogeneous networks.

\section{Resource partitioning in macrocell-femtocell networks}

In this study, the goal of resource partitioning is to split the total bandwidth $B$ among tiers such that (1) The capacity of the overall system is maximized, (2) a level of global fairness is ensured between users in different tiers, and (3) QoS requirements of users in different tiers in terms of relative data rates are satisfied.

As shown in Figure $1 \mathrm{~b}$, the portion of the accessed bandwidth for macrocell-tier is $\rho$ where $\rho=\frac{B_{\mathrm{M}}}{B}$. Therefore, $B_{\mathrm{F}}=(1-\rho) B$ where $B_{\mathrm{M}}=\sum_{k=1}^{N_{\mathrm{U}, 1,1}} B_{1,1, k}$ and $B_{\mathrm{F}}=\sum_{k=1}^{N_{\mathrm{U}, 2, j}} B_{2, j, k}, \forall j$. In addition, in the hybrid approach, Figure 1c, the portion of the accessed bandwidth for macrocell-tier is the total bandwidth $B_{\mathrm{M}}=$ $B$, and $B_{\mathrm{F}}=(1-\rho) B$ where $B_{\mathrm{M}}=\sum_{k=1}^{N_{\mathrm{U}, 1,1}} B_{1,1, k}$ and $B_{\mathrm{F}}=\sum_{k=1}^{N_{\mathrm{U}, 2, j}} B_{2, j, k}, \quad \forall j$. In both approaches, our goal when splitting the spectrum is to maximize $C_{\text {Tot }}(\rho)=\sum_{i=1}^{T} \sum_{j=1}^{N_{\mathrm{N}, i}} \sum_{k=1}^{N_{\mathrm{U}, i, j}} C_{i, j, k}(\rho)$ while considering fairness metric and QoS parameter.

\subsection{Macrocell and femtocell deployment using HPP}

In this section, we focus on a general analytical formulation of the macrocell-femtocell capacities by employing statistical models for $\mathrm{mBS}$ and fBS locations ${ }^{\mathrm{d}}$. We focus on an arbitrary mobile station (MS) in this region and calculate the downlink capacity for macrocell and femtocell users for dedicated channel, co-channel, and hybrid channel scenarios. These results, which are functions only of the macrocell and femtocell relative densities, transmit powers, the parameters of the wireless channel, and the SSR, and provide valuable insights for the architecture planning process for joint femtomacro deployments under different fairness and QoS criteria. 
The mBS locations are assumed to be points of a HPP on the plane with intensity $\lambda$ :

1. The number of $\operatorname{mBS} N(\mathbb{B})$ in any finite region $\mathbb{B}$ is Poisson $(\lambda \times \operatorname{area}(\mathbb{B}))$ : for $n=0,1, \cdots$,

$$
\mathbb{P}\{N(\mathbb{B})=n\}=\mathrm{e}^{-\lambda \times \operatorname{area}(\mathbb{B})} \frac{[\lambda \times \operatorname{area}(\mathbb{B})]^{n}}{n !}
$$

with mean $\mathbb{E} N(\mathbb{B})=\lambda \times \operatorname{area}(\mathbb{B})$.

2. $\forall \mathbb{B}, \mathbb{B}^{\prime}: \mathbb{B} \cap \mathbb{B}^{\prime}=\varnothing \Rightarrow N(\mathbb{B}), N\left(\mathbb{B}^{\prime}\right)$ are independent.

3. $\forall \mathbb{B}$, given $N(\mathbb{B})=n$, these $n$ mBS are i.i.d and uniformly distributed over $\mathbb{B}$.

Note that $\lambda$ is in units of points per square meter. We model the locations of fBS by points of an independent HPP with intensity $\lambda^{\prime}$ and all the fBSs that are operating in CSG mode.

The wireless channel model we use in this study can be defined by the following assumptions:

1. Path loss exponent is $\delta$.

2. We assume no shadowing in our analysis, purely for reasons of analytical tractability as discussed in [17].

3. Fading in all macrocellular downlinks are independent identically distributed (i.i.d.) Rayleigh with mean $1^{\mathrm{e}}$.

4. All mBS (respectively fBS) transmit with the same reference symbol power $P_{\mathrm{RS}}$ (respectively $P_{\mathrm{RS}}^{\prime}$ ).

5. MS at distance $r$ from $\mathrm{mBS}$ has a reference symbol received power (RSRP in long-term evolution (LTE) terminology)

$$
\operatorname{RSRP}(r)=\frac{H}{r^{\delta}}, H \sim \exp (P), P \equiv K P_{\mathrm{RS}},
$$

where the exponential distribution of $H$ arises from the Rayleigh fading assumption, and $K$ is a quantity that takes into account the relative heights of the transmitter and receiver on the link, etc., and is considered the same for all links from any MS location to any mBS. We are interested in MS locations whose distance from the nearest $\mathrm{mBS}$ exceeds some $r_{\text {min }}$.

6. Similarly, a MS at distance $r^{\prime}$ from a fBS has RSRP given by

$$
\operatorname{RSRP}^{\prime}\left(r^{\prime}\right)=\frac{H^{\prime}}{r^{\prime} \delta}, H^{\prime} \sim \exp \left(P^{\prime}\right), P^{\prime} \equiv K^{\prime} P_{\mathrm{RS}}^{\prime} .
$$

Theorem Suppose that there are transmitters located at points of $M$ independent HPPs $1, \ldots, M$, with intensities $v_{1}, \cdots, v_{M}$, respectively, and that the MS must be a minimum distance of $d_{\mathrm{min}, k}$ from the nearest transmitter of HPP $k, k=1, \cdots, M$. The fading coefficients on all transmitter-MS links are independent, and those on the links between the MS and the transmitters belonging to $H P P k$ are i.i.d. $\exp (\mu k), k=1, \cdots, M$. Suppose the MS is at a distance of $r$ from the nearest transmitter of HPP
1, then the complementary cumulative distribution function of signal interference ratio, $\operatorname{SIR}(r)$, which is the SIR at the MS when served by the nearest transmitter of HPP 1 , is given by

$$
\begin{aligned}
& \mathbb{P}\left\{\Gamma_{1}>\gamma \mid R_{1}=r_{1}\right\} \\
& \quad=\exp \left(-u \gamma^{\frac{2}{\delta}}\left[G\left(\frac{1}{\gamma^{\frac{2}{\delta}}}\right)+\sum_{k=2}^{M} \Theta_{k} G\left(\frac{m_{k}}{u \gamma^{\frac{2}{\delta}} \Theta_{k}}\right)\right]\right),
\end{aligned}
$$

where $u=v_{1} \pi r_{1}^{2}$ and for $k=1, \ldots, M$,

$$
\begin{gathered}
m_{k}=v_{k} \pi d_{\min , k}^{2}, \quad \Theta_{k}=\frac{v_{k}}{v_{1}}\left(\frac{\mu_{k}}{\mu_{1}}\right)^{2 / \delta} \\
G(y)=\int_{y}^{\infty} \frac{\mathrm{d} x}{1+x^{\frac{\delta}{2}}}=\left\{\begin{array}{cc}
\pi / 2-\tan ^{-1} y & \delta=4 \\
\left.{ }_{2} F_{1}\left(1, \frac{\delta}{2} ; 1+\frac{\delta}{2} ;-x^{\frac{\delta}{2}}\right) x\right|_{y} ^{\infty} & \delta \neq 4
\end{array},\right.
\end{gathered}
$$

and ${ }_{2} F_{1}(a, b ; c ; z)$ is the hypergeometric function

$$
{ }_{2} F_{1}(a, b ; c ; z)=1+\sum_{n=1}^{\infty} \frac{z^{n}}{n !} \prod_{l=0}^{n-1} \frac{(a+l)(b+l)}{c+l} .
$$

Proof. [18] To provide the mean capacity, we use the signal to interference plus noise ratio (SINR) $(\Gamma)$ distribution ${ }^{f}$ (13) in macrocell and femtocell tier capacities provided in (2) and (3). The mean rate of MS at distance $R_{1}=r_{1}$ from nearest BS of HPP 1 can be given as

$$
\begin{aligned}
C_{1}\left(r_{1}\right) & =\mathbb{E}_{\Gamma_{1}}\left[\log _{2}\left(1+\Gamma_{1}\left(r_{1}\right)\right)\right] \\
& =\int_{0}^{\infty} \mathbb{P}\left\{\Gamma_{1}>2^{x}-1 \mid R_{1}=r_{1}\right\} \mathrm{d} x .
\end{aligned}
$$

Let $\lambda_{\mathrm{MS}, 1}$ be the density of the MSs for the HPP 1 . Then the aggregate rate over the region served by a single $\mathrm{BS}$ of HPP 1 can be written as

$$
\begin{aligned}
C_{1, \mathrm{BS}} & =\int_{d_{\min , 1}}^{d_{\mathrm{max}, 1}} 2 \pi r_{1} \lambda_{\mathrm{MS}, 1} C_{1}\left(r_{1}\right) \mathrm{d} r_{1} \\
& =\int_{d_{\mathrm{min}, 1}^{2}}^{d_{\mathrm{max}, 1}^{2}} \pi \lambda_{\mathrm{MS}, 1} \tilde{C}_{1}(t) \mathrm{d} t
\end{aligned}
$$

using the change of variable $t=r_{1}^{2}$. Therefore using (13), $\tilde{C}(t)$ can be given as

$$
\begin{aligned}
\tilde{C}(t)=\int_{0}^{\infty} \exp \left(-v_{1} \pi t\left(2^{x}-1\right)^{\frac{2}{\delta}}\left[G\left(\frac{1}{\left(2^{x}-1\right)^{\frac{2}{\delta}}}\right)\right.\right. \\
\left.\left.+\sum_{k=2}^{M} \Theta_{k} G\left(\frac{m_{k}}{v_{k} \pi t\left(2^{x}-1\right)^{\frac{2}{\delta}} \Theta_{k}}\right)\right]\right) \mathrm{d} x .
\end{aligned}
$$


Then, the capacity for a given tier can be written in a generalized form as follows:

$$
\begin{aligned}
C_{i} & =\frac{B N_{\mathrm{N}, i}}{\pi \lambda_{\mathrm{MS}, 1}\left(d_{\mathrm{max}, 1}^{2}-d_{\mathrm{min}, 1}^{2}\right)} \int_{d_{\mathrm{min}, 1}^{2}}^{d_{\max , 1}^{2}} \pi \lambda_{\mathrm{MS}, 1} \tilde{C}(t) \mathrm{d} t \\
& =\mathbb{C}\left(B, N_{\mathrm{N}, i}, M, \boldsymbol{v}, \boldsymbol{\mu}, \boldsymbol{m}, \boldsymbol{\Theta}, d_{\mathrm{max}, 1}, d_{\min , 1}\right)
\end{aligned}
$$

where the total bandwidth is assumed to be distributed in a round-robin fashion in each $\mathrm{mBS}$ and $\mathrm{fBS}$ with an assumption that all of the users are continuously backlogged. Note that $\boldsymbol{v}=\left[v_{1}, \ldots, v_{M}\right], \boldsymbol{\mu}=\left[\mu_{1}, \ldots, \mu_{M}\right]$, $\boldsymbol{m}=\left[m_{1}, \ldots, m_{M}\right]$, and $\boldsymbol{\Theta}=\left[\Theta_{1}, \ldots, \Theta_{M}\right]$ are vectors of $1 \times M$, with $m_{k}$ and $\Theta_{k}$ as in (14). For a given area with radius $R$, the number of macrocells and femtocells can be calculated as $N_{\mathrm{N}, 1}=\lambda \pi R^{2}$ and $N_{\mathrm{N}, 2}=\lambda^{\prime} \pi R^{2}$, respectively.

\subsection{Co-channel macrocell/femtocell networks}

By using (2) and the same notation, the capacity of femtocell tier can be written as

$$
C_{\mathrm{Fem}}=\frac{B}{N_{\mathrm{U}, \mathrm{F}}} \mathbb{E}\left[\sum_{j=1}^{N_{\mathrm{N}, 2}} \sum_{k=1}^{N_{\mathrm{U}, \mathrm{F}}} \log _{2}\left(1+\Gamma_{j, k}\right)\right],
$$

where $B_{2, j, k}=\frac{B_{\mathrm{F}}}{N_{\mathrm{U}, 2, j}}=\frac{B}{N_{\mathrm{U}, \mathrm{F}}}, \forall j, k$. Then using (20) we can rewrite (21) as

$$
C_{\mathrm{Fem}}=\mathbb{C}\left(B, N_{\mathrm{N}, 2}, M, \boldsymbol{v}, \boldsymbol{\mu}, \boldsymbol{m}, \boldsymbol{\Theta}, d_{\mathrm{max}, 1}, d_{\mathrm{min}, 1}\right)
$$

where $M=2$; HPP1 is fBS locations; HPP2 is mBS locations $\left(v_{1}, v_{2}\right)=\left(\lambda^{\prime}, \lambda\right),\left(\mu_{1}, \mu_{2}\right)=\left(P^{\prime}, P\right)$, $\left(m_{1}, m_{2}\right)=\left(\lambda^{\prime} \pi r_{\min , \mathrm{f}}^{2} \lambda \pi r_{\min , \mathrm{m}}^{2}\right), \Theta_{2}=\frac{\lambda}{\lambda^{\prime}}\left(\frac{P}{P^{\prime}}\right)^{2 / \delta}$, and $\left(d_{\text {max }, 1}, d_{\text {min, } 1}\right)=\left(r_{\text {max }, \mathrm{f}}, r_{\text {min,f }}\right)$. Note that $r_{\text {min,m }}$ and $r_{\text {min, }}$ are the minimum distances between a MS-mBS, and MS$\mathrm{fBS}$, respectively; and $r_{\text {max,m }}$ and $r_{\text {max,f }}$ are the maximum distances between a MS-mBS, and MS-fBS, respectively. Similarly, using (3)

$$
C_{\mathrm{Mac}}=\frac{B}{N_{\mathrm{U}, \mathrm{M}}} \mathbb{E}\left[\sum_{j=1}^{N_{\mathrm{N}, 1}} \sum_{k=1}^{N_{\mathrm{U}, \mathrm{M}}} \log _{2}\left(1+\Gamma_{j, k}\right)\right],
$$

where $B_{2, j, k}=\frac{B_{\mathrm{F}}}{N_{\mathrm{U}, 2, j}}=\frac{B}{N_{\mathrm{U}, \mathrm{F}}}, \forall j, k$. Then using (20) we can rewrite (23) as

$$
C_{\mathrm{Mac}}=\mathbb{C}\left(B, N_{\mathrm{N}, 1}, M, \boldsymbol{v}, \boldsymbol{\mu}, \boldsymbol{m}, \boldsymbol{\Theta}, d_{\mathrm{max}, 1}, d_{\mathrm{min}, 1}\right)
$$

where $M=2$; HPP1 is mBS locations; HPP2 is fBS locations $\left(\nu_{1}, v_{2}\right)=\left(\lambda, \lambda^{\prime}\right),\left(\mu_{1}, \mu_{2}\right)=\left(P, P^{\prime}\right)$, $\left(m_{1}, m_{2}\right)=\left(\lambda \pi r_{\text {min, }}^{2}, \lambda^{\prime} \pi r_{\text {min, }}^{2}\right), \Theta_{2}=\frac{\lambda^{\prime}}{\lambda}\left(\frac{P^{\prime}}{P}\right)^{2 / \delta}$, and $\left(d_{\max , 1}, d_{\min , 1}\right)=\left(r_{\max , \mathrm{m}}, r_{\min , \mathrm{m}}\right)$.

\subsection{Dedicated channel macrocell/femtocell networks}

Using (2) and the same notation, the capacity of femtocell tier can be written as

$$
C_{\mathrm{Fem}}(\rho)=\frac{B(1-\rho)}{N_{\mathrm{U}, \mathrm{F}}} \mathbb{E}\left[\sum_{j=1}^{N_{\mathrm{N}, 2}} \sum_{k=1}^{N_{\mathrm{U}, \mathrm{F}}} \log _{2}\left(1+\Gamma_{j, k}\right)\right] .
$$

Then using (20) we can rewrite (25) as

$$
C_{\mathrm{Fem}}(\rho)=\mathbb{C}\left(B(1-\rho), N_{\mathrm{N}, 2}, M, v, \mu, m, 0, d_{\mathrm{max}, 1}, d_{\mathrm{min}, 1}\right),
$$

where $M=1$; HPP1 is fBS locations $v_{1}=\lambda^{\prime}, \mu_{1}=P^{\prime}$, $m_{1}=\lambda^{\prime} \pi r_{\min , \mathrm{f}}^{2}$, and $\left(d_{\max , 1}, d_{\min , 1}\right)=\left(r_{\max , \mathrm{f}}, r_{\min , \mathrm{f}}\right)$. Similarly, using (3)

$$
C_{\mathrm{Mac}}(\rho)=\frac{B \rho}{N_{\mathrm{U}, \mathrm{M}}} \mathbb{E}\left[\sum_{j=1}^{N_{\mathrm{N}, 1}} \sum_{k=1}^{N_{\mathrm{U}, \mathrm{M}}} \log _{2}\left(1+\Gamma_{j, k}\right)\right] .
$$

Then using (20) we can rewrite (27) as

$$
C_{\mathrm{Mac}}(\rho)=\mathbb{C}\left(B \rho, N_{\mathrm{N}, 1}, M, v, \mu, m, 0, d_{\mathrm{max}, 1}, d_{\mathrm{min}, 1}\right),
$$

where $M=1$; HPP1 is mBS locations, $v_{1}=\lambda, \mu_{1}=P$, $m_{1}=\lambda \pi r_{\min , \mathrm{m}}^{2}$, and $\left(d_{\max , 1}, d_{\min , 1}\right)=\left(r_{\max , \mathrm{m}}, r_{\mathrm{min}, \mathrm{m}}\right)$.

Therefore, the total capacity for the dedicated channel scenario using (26) and (28) can be given as $C_{\text {Tot }}(\rho)=$ $C_{\mathrm{Mac}}(\rho)+C_{\mathrm{Fem}}(\rho)$. Then, spectrum splitting $\rho$ value that maximizes the $C_{\text {Tot }}(\rho)$ in can be expressed as follows:

$$
\rho_{\max }=\arg \max _{0 \leq \rho \leq 1} C_{\text {Tot }}(\rho) .
$$

Note that the objective function in (29) is a linear combination of (26) and (28). Since each femtocell reuses the spectrum more frequently, the capacity equation given in (26) includes a larger multiplying term $N_{\mathrm{N}, 2}>N_{\mathrm{N}, 1}$. Therefore, if the SINR levels of users in each tier are similar, objective function (29) will be maximized at $\rho=0$. This issue is also investigated by calculating per-tier area spectral efficiencies (ASEs) in [19] and it is shown that capacity is maximized at extreme points without a fairness or QoS parameter. Such a partitioning is obviously unfair since it results in a greedy allocation to one of the tiers which will be discussed in more detail in Section 4.2.

\subsection{Hybrid approach for resource allocation}

In this section, we investigate the hybrid approach scenario as it is shown in Figure 1c. Using (2), the total capacity of femtocell network can be calculated with a slight modification of (22), where the bandwidth of femtocell is $\rho B$, and femtocell users are always co-channelled with macrocell, that is,

$$
C_{\mathrm{Fem}}(\rho)=\mathbb{C}\left(B(1-\rho), N_{\mathrm{N}, 2}, M, \boldsymbol{v}, \boldsymbol{\mu}, \boldsymbol{m}, \boldsymbol{\Theta}, d_{\mathrm{max}, 1}, d_{\mathrm{min}, 1}\right),
$$


where $M=2$; HPP1 is fBS locations; HPP2 is mBS locations $\left(v_{1}, \nu_{2}\right)=\left(\lambda^{\prime}, \lambda\right),\left(\mu_{1}, \mu_{2}\right)=\left(P^{\prime}, P\right)$, $\left(m_{1}, m_{2}\right)=\left(\lambda^{\prime} \pi r_{\text {min, }}^{2}, \lambda \pi r_{\text {min,m }}^{2}\right), \Theta_{2}=\frac{\lambda}{\lambda^{\prime}}\left(\frac{P}{P^{\prime}}\right)^{2 / \delta}$, and $\left(d_{\max , 1}, d_{\min , 1}\right)=\left(r_{\max , \mathrm{f}}, r_{\min , \mathrm{f}}\right)$. Note that $r_{\min , \mathrm{m}}$ and $r_{\min , \mathrm{f}}$ are the minimum distances between a MS-mBS and MSfBS, respectively, and note that $r_{\max , \mathrm{m}}$ and $r_{\text {max,f }}$ are the maximum distances between a MS-mBS, and MS-fBS, respectively.

On the other hand, to calculate the macrocell capacity, the following steps should be followed:

- Consider a macrocell MS at distance $r_{1}$ from its nearest $\mathrm{mBS}$. Let $\gamma_{c}$ be the minimum rate for scheduling a mMS to dedicated channel portion of hybrid channel. Then the instantaneous rate of this MS from the MBS can be given as

$$
\begin{aligned}
C_{\text {macro }}\left(r_{1}\right) & =(1-\rho) C_{\mathrm{co}}\left(r_{1}\right) 1\left\{C_{\mathrm{co}}\left(r_{1}\right)>\gamma_{c}\right\} \\
& +\rho C_{\mathrm{ded}}\left(r_{1}\right) 1\left\{C_{\mathrm{co}}\left(r_{1}\right) \leq \gamma_{c}\right\} .
\end{aligned}
$$

Note that $C_{\mathrm{co}}\left(r_{1}\right), C_{\mathrm{ded}}\left(r_{1}\right)$ are instantaneous (includes effects of fading) rates derived in (17) for co-channel and dedicated channel scenarios ${ }^{\mathrm{g}}$, respectively.

- Therefore, the mean rate for that MS can be given as

$$
\begin{aligned}
\bar{C}_{\text {macro }}\left(r_{1}\right) & =(1-\rho) \mathbb{E}\left[C_{\mathrm{co}}\left(r_{1}\right) 1\left\{C_{\mathrm{Co}}\left(r_{1}\right)>\gamma_{c}\right\}\right] \\
& +\rho \mathbb{E}\left[C_{\text {ded }}\left(r_{1}\right) 1\left\{C_{\mathrm{co}}\left(r_{1}\right) \leq \gamma_{c}\right\}\right]
\end{aligned}
$$

- Finally, the aggregate rate for an mBS can be written as

$$
\begin{aligned}
C_{\mathrm{Mac}}(\rho)= & \frac{B N_{\mathrm{N}, 1}}{\pi \lambda_{\mathrm{MS}, 1}\left(d_{\max , 1}^{2}-d_{\mathrm{min}, 1}^{2}\right)} \\
& \times \int_{d_{\mathrm{min}, 1}}^{d_{\max , 1}} 2 \pi \lambda_{\mathrm{MS}, 1} r_{1} \bar{C}_{\mathrm{macro}}\left(r_{1}\right) \mathrm{d} r_{1} .
\end{aligned}
$$

Therefore, the macro-tier capacity can be provided as in (35), by solving for (33) and (34). Let $X=$ $C_{\mathrm{co}}\left(r_{1}\right) 1\left\{C_{\mathrm{co}}\left(r_{1}\right)>\gamma_{c}\right\}$. Then,

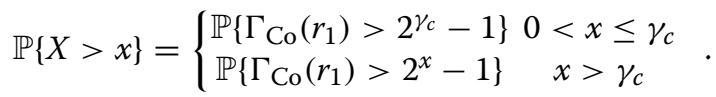

Therefore,

$$
\begin{aligned}
\mathbb{E}[X]= & \int_{0}^{\infty} \mathbb{P}\{X>x\} \mathrm{d} x \\
= & \gamma_{c} \underbrace{\int_{\gamma_{c}}^{\infty} \mathbb{P}\left\{\Gamma_{\mathrm{Co}}\left(r_{1}\right)>2^{x}-1\right\} \mathrm{d} x}_{\text {substitute } \gamma=2^{\gamma_{c}-1 \text { in (13) }} \mathbb{P}\left\{\Gamma_{\mathrm{Co}}\left(r_{1}\right)>2^{\gamma_{c}}-1\right\}} . \\
& \underbrace{\int_{17}}_{\text {integral in (17) with } \gamma_{c}}
\end{aligned}
$$

To calculate (34), we assume that the interference from indoor femtocells to outdoor macrocell MSs is negligible due to the low power of femtocells and wall loss; therefore, $C_{\text {ded }}\left(r_{1}\right) \simeq C_{\text {co }}\left(r_{1}\right)$. Then

$$
\begin{aligned}
\mathbb{E} & {\left[C_{\mathrm{ded}}\left(r_{1}\right) 1\left\{C_{\mathrm{co}}\left(r_{1}\right) \leq \gamma_{c}\right\}\right] } \\
& \simeq \mathbb{E}\left[C_{\mathrm{co}}\left(r_{1}\right)\left(1-1\left\{C_{\mathrm{co}}\left(r_{1}\right)>\gamma_{c}\right\}\right)\right] \\
& =\mathbb{E}\left[C_{\mathrm{co}}\left(r_{1}\right)\right]-\underbrace{\mathbb{E}\left[C_{\mathrm{co}}\left(r_{1}\right)\left(1\left\{C_{\mathrm{co}}\left(r_{1}\right)>\gamma_{c}\right\}\right)\right]}_{\mathbb{E}[X]}
\end{aligned}
$$

Therefore, (35) can be calculated with

$$
\begin{aligned}
\bar{C}_{\text {macro }}\left(r_{1}\right)= & (1-\rho) \mathbb{E}\left[C_{\mathrm{co}}\left(r_{1}\right) 1\left\{C_{\mathrm{co}}\left(r_{1}\right)>\gamma_{c}\right\}\right] \\
& +\rho \mathbb{E}\left[C_{\mathrm{ded}}\left(r_{1}\right) 1\left\{C_{\mathrm{co}}\left(r_{1}\right) \leq \gamma_{c}\right\}\right] \\
= & (1-2 \rho) \mathbb{E}[X]+\rho \mathbb{E}\left[C_{\mathrm{co}}\left(r_{1}\right)\right] .
\end{aligned}
$$

Therefore, the total capacity for the hybrid channel scenario using (30) and (35) can be given as $C_{\text {Tot }}(\rho)=$ $C_{\text {Mac }}(\rho)+C_{\text {Fem }}(\rho)$. Then, the spectrum splitting $\rho$ value that maximizes the $C_{\text {Tot }}(\rho)$ can be expressed as follows:

$$
\rho_{\max }=\arg \max _{0 \leq \rho \leq 1} C_{\text {Tot }}(\rho) .
$$

In hybrid channel scenario, jointly choosing $\gamma_{c}$ and $\rho$ becomes important since different sum capacities and fairness levels could be achieved with these two values. If the macrocell users which have lower SINRs can be scheduled to the dedicated channel portion, there will be no victim users. Therefore, the number of users assigned to dedicated channel should be selected carefully since the amount of bandwidth corresponding to dedicated channel should be kept less in order to fully utilize the resource in both tiers. First, the macrocell MSs should be sorted with respect to their maximum achievable co-channel capacities, and, second, $N_{\mathrm{U}, \mathrm{M}}^{(\rho)}$ macrocell MSs with worse capacities should be scheduled in the dedicated channel portion (i.e., $N_{\mathrm{U}, \mathrm{M}}^{(1-\rho)}$ mMS scheduled in co-channel portion). Note that $N_{\mathrm{U}, \mathrm{M}}^{(\rho)}$ and $\gamma_{c}$ has a direct relation, that is, $\gamma_{c}$ determines how many users will be assigned to dedicated channel portion $N_{\mathrm{U}, \mathrm{M}}^{(\rho)}$. However, it is also important to note that the value of SSR $(\rho)$ determines the bandwidth to be assigned for each user depending on co-channel or dedicated channel spectrum. Although analytical derivations provide the intuition between these relationships with (35) and (39), we investigate the optimum solution of this problem in details in our computer simulations. In our simulations, while selecting $N_{\mathrm{U}, \mathrm{M}}^{(\rho)}$, we consider a maxmin scheduler that maximizes the minimum capacity of macrocell users as follows [20]:

$$
N_{\mathrm{U}, \mathrm{M}}^{(\rho)}=\arg \max _{N_{\mathrm{U}, \mathrm{M}}^{(\rho)}}\left\{\min _{k} C_{1,1, k}\right\}
$$




$$
\rho=\arg \max _{0 \leq \rho \leq 1}\left\{C_{\mathrm{Tot}}\left(\rho, N_{\mathrm{U}, \mathrm{M}}^{(\rho)}\right)\right\} .
$$

By using simple max-min capacity scheduling, the minimum capacity of mMS are maximized by assigning them to dedicated channel portion while also maximizing the overall capacity of the macrocell-femtocell network. The fairness and QoS orientation constraints in the network can also be introduced by usage of fairness metric given in (10).

\section{Numerical results}

In this section, the numerical results for both analytical derivations and simulations are presented. First, we investigate the behavior of the discussed fairness indices for a particular scenario. Then, we present the optimum spectrum splitting strategy based on computer simulations and analytical derivations for co-channel, dedicated channel, and hybrid approach scenarios. Mathematical modeling is shown to be aligned with simulation results when the basic simulation parameters shown in Table 2 are used. Finally, to investigate the details of all approaches in further detail, a 3GPP compatible simulator using the parameters given in [21] is used together with a max-min fair scheduler for hybrid approach. Some of the critical parameters used for 3GPP aligned simulations are also summarized in Table 2.

\subsection{Comparison of different fairness metrics}

The effect of the number of cells and the number of users in each cell with the bounds in Table 1 is investigated in a two-tier network case study $(T=2)$, where tier- 1 has one cell $\left(N_{\mathrm{N}, 1}=1\right)$ and tier-2 has two cells $\left(N_{\mathrm{N}, 2}=2\right)$. We consider two different scenarios to provide a better understanding for the metrics and their related bounds. In the first scenario, we assume that there are $N_{\mathrm{U}, 1,1}=4$ users in tier-1, cell-1, and $N_{\mathrm{U}, 2,1}=3 ; N_{\mathrm{U}, 2,2}=1$ users for tier- 2 and cells 1 and 2 . Therefore, there are a total of eight users in the network for the first scenario. In the second scenario, we do not change the total number of users; however, we consider $N_{\mathrm{U}, 1,1}=6$ users in tier-1, cell-1, and $N_{\mathrm{U}, 2,1}=1$ and $N_{\mathrm{U}, 2,2}=1$ users for tier- 2 and cells 1 and 2 .

The allocated resources for each user is assumed to be partitioned in a round-robin fashion within each cell in all tiers, and the capacity of each cell is normally distributed with mean $\mu$ and variance $\sigma^{2}\left(C_{i, j} \sim \mathcal{N}\left(\mu, \sigma^{2}\right)\right)$. Figure 2 shows that proposed fairness index (TFI) is between $\left[1 / N_{\text {Tot }}, 1\right]$ with controlled boundings, converging to WJFI at 1 for small standard deviation values. On the other hand, TFI converges to JFI at 0.125 for increasing standard deviation. Moreover, upper bound of JFI is decreased and lower bound of WJFI is increased in scenario 2 compared to scenario 1 . The non-even distribution of the users in cells increases the lower bound of the WJFI. For instance in scenario 2, the cells 1 and 2 in tier 2

Table 2 Numerical parameters for analytical/simulation results

\begin{tabular}{|c|c|c|}
\hline \multirow[t]{2}{*}{ Parameter } & \multicolumn{2}{|c|}{ Description/value } \\
\hline & Analytical results and basic simulator & 3GPP compatible simulator [21] \\
\hline Macrocellular & Hexagonal layout with BS in the middle of the cell & Hexagonal layout with cell-center BSs \\
\hline Number of mBS & $\begin{array}{l}\text { Infinite for analytical, } 19 \text { cell with wrap around for } \\
\text { simulations }\end{array}$ & 19 cell with wrap around \\
\hline Inter-mBS distance & $\begin{array}{l}500 \mathrm{~m} \text { for simulations; } 500 \mathrm{~m} \text { in average for analyti- } \\
\text { cal. Therefore, the density of the macrocells }\left(\lambda_{\mathrm{mBS}}\right) \\
\text { is } \frac{1}{500 \sqrt{3} / 2}=4.62 \times 10^{-6} \text {. Similarly, the density of } \\
\text { femtocells is } \lambda_{\mathrm{fBS}}=\lambda_{\mathrm{mBS}} \times 12=5.54 \times 10^{-5}\end{array}$ & $500 \mathrm{~m}$ \\
\hline Number of fBS & 12 per each macrocell & 12 per each macrocell \\
\hline fBs distribution & $\begin{array}{l}12 \text { fBSs that are randomly and uniformly dis- } \\
\text { tributed within each sector in CSG mode }\end{array}$ & $\begin{array}{l}4 \mathrm{fBSs} \text { that are randomly and uniformly dis- } \\
\text { tributed within each sector in CSG mode }\end{array}$ \\
\hline mBS-mMS minimum distance & $35 \mathrm{~m}$ & $35 \mathrm{~m}[21]$ \\
\hline fBS-fMS minimum distance & $5 \mathrm{~m}$ & $5 \mathrm{~m}[21]$ \\
\hline Bandwidth & $10 \mathrm{MHz}$ & $10 \mathrm{MHz}$ \\
\hline $\mathrm{DL}$ transmit power mBS & $60 \mathrm{dBm}$ & $\begin{array}{l}46 \mathrm{dBm} \text {, with } \mathrm{Tx} \text { power at } \mathrm{mBS} \text { and } 14 \mathrm{dBi} \\
\text { antenna gain [21]. Three sectors with } 3-\mathrm{D} \\
\text { antenna pattern. Antenna height, } 32 \mathrm{~m}\end{array}$ \\
\hline DL transmit power fBS & $20 \mathrm{dBm}$ & $20 \mathrm{dBm}$ with antenna gain of $5 \mathrm{dBi}$ \\
\hline Thermal noise density & $-174 \mathrm{dBm} / \mathrm{Hz}$ & $-174 \mathrm{dBm} / \mathrm{Hz}$ \\
\hline Path loss model (macrocell) & $128.1+40 \log _{10}(R), R$ in $\mathrm{km}$ & $128.1+37.6 \log _{10}(R), R$ in $\mathrm{km}[21]$ \\
\hline Path loss model (femtocell) & $127+40 \log _{10}(R), R$ in km & $127+36.7 \log _{10}(R), R$ in km [21] \\
\hline Wall loss attenuation & $20 \mathrm{~dB}$ & $20 \mathrm{~dB}$ \\
\hline
\end{tabular}


have only one user. Calculating the lower bound of WJFI according to Table 1 for scenario 1 and scenario 2 provides 0.527 and 0.722 , respectively, which could also be tracked from Figure 2. Although an upper bound independent from allocated resource could not be achieved for JFI, Figure 2 shows that while the number of users in a cell (for instance, the number of users in tier 1 cell 1 is very high compared to tier 2 cells) increases, the upper bound decreases.

\subsection{Numerical results for analytical derivations}

In this section, we present the numerical results for equations derived through Equations 21 to 39. A computer simulation is demonstrated to provide a comparison between analytical derivations and simulation results. The simulation scenario includes analytical derivation assumptions and uses the parameters listed in Table 1.

Figure 3 shows the sum capacity of a macrocell for different SSR values for co-channel, dedicated channel, and hybrid approaches. Results show that co-channel scenario has better capacity when compared with dedicated and hybrid channel approaches for macrocell sum capacities. While $\rho$ is increasing, the bandwidth assigned to macrocell users is increasing and at $\rho=1$, the capacity of dedicated channel is almost same with co-channel approach. This result shows that for the given scenario, CSG indoor femtocell BSs are not causing severe interference to macro MSs; therefore, co-channel scenario outperforms the dedicated channel scenario. On the other hand, for hybrid channel scenario, for the given $\gamma_{c}=10^{4} \mathrm{bps}$, increasing SSR decreases the macrocell capacity. For a fixed $\gamma_{c}$ value, the number of users assigned to dedicated channel portion is fixed and those users are the ones that has the lowest capacity. Therefore, increasing the dedicated channel portion with increasing $\rho$ decreases the capacity, since the bandwidth assigned to a small number of users which has lower SINRs decreases the sum capacity in a macrocell. In extreme cases of $\rho=0,1$, the hybrid approach converges to co-channel and dedicated channel approaches. It is also important to note that simulation results and analytical results are aligned.

Figure 4 shows the sum capacity of a femtocell for different SSR values for co-channel, dedicated channel, and hybrid approaches. The co-channel capacity of a femtocell is greater than dedicated channel for larger values of SSR, where femtocell bandwidth is less. Note that similar capacities can be achieved with co-channel and dedicated channel approaches for $\rho \simeq 0.75$. On the other hand, increasing SSR for hybrid channel scenario also decreases the sum capacity of femtocells converging to co-channel at $\rho=0$ and dedicated channel at $\rho=1$. As a result, it can be concluded that for a fixed $\gamma_{c}$, and without fairness and QoS constraints, resource partitioning cannot be done effectively since extreme points are maximizing the capacity for both macrocells and femtocells.

\subsection{Detailed investigation of computer simulations and max-min scheduling under fairness and QoS constraints}

Previous section shows that although computer simulations and analytical results are aligned, the sum capacity is maximized at extreme points for both macrocell and femtocell networks. Therefore, in this section, we introduce the fairness criterion into the optimization while also considering a more applicable scenario where parameters are selected from [21]. This study also considers the

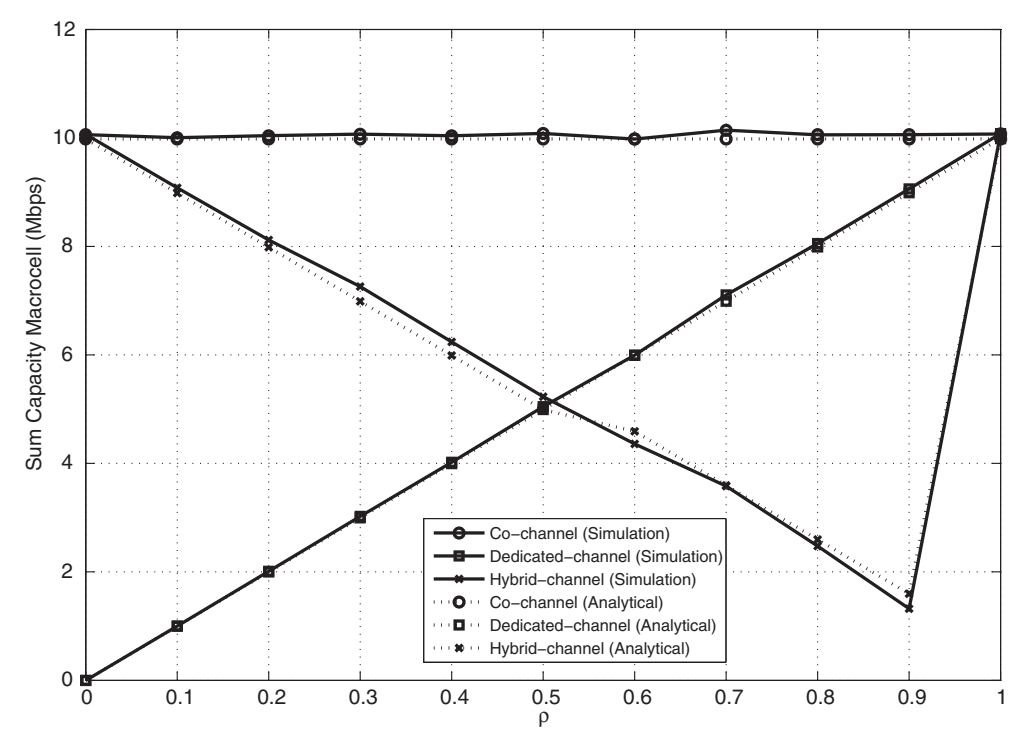

Figure 3 Sum capacity of macrocell users vs. SSR $\rho$. 


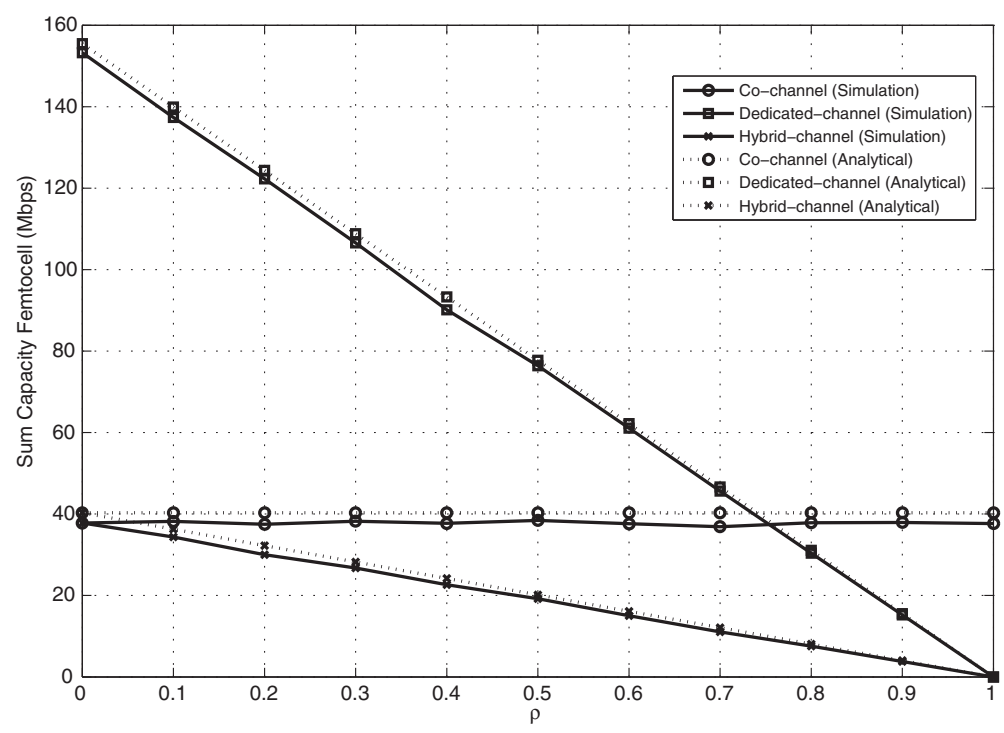

Figure 4 Sum capacity of femtocell users vs. SSR $\rho$.

case where a portion of macrocell MSs is inside the CSG femtocell area which we called indoor ratio (IR). One hundred users are randomly and uniformly distributed within each sector, and there are two users associated with each closed-access femtocell [21]. This yields $100-4 \times 2=92$ macrocell users within each sector.

In Figure 5, SINR CDFs for the co-channel scenario with IR $=0$, dedicated channel scenario, and hybrid channel scenarios SINRs are aligned with the 3GPP benchmarks in [21]. In co-channel scenario, for larger IR, SINRs of victim macrocell MSs get worse due to increasing interference. On the other hand, since dedicated channel approach uses separate bandwidths for macrocell and femtocell, such behavior is not observed. It is also important to note that if $\mathrm{IR}=0$, the co-channel and dedicated channel SINRs are same, which validates the assumption in (38) for CSG scenario with wall loss. Moreover, the hybrid approach with max-min fair scheduler also protects the victim macro MSs by assigning them to the dedicated portion of hybrid approach.

In Figures 6 and 7, the sum capacity of a macrocell and 5-percentile capacity of macrocell MSs are provided for various IR and SSR, respectively. Note that in both figures, the hybrid approach converges to co-channel at

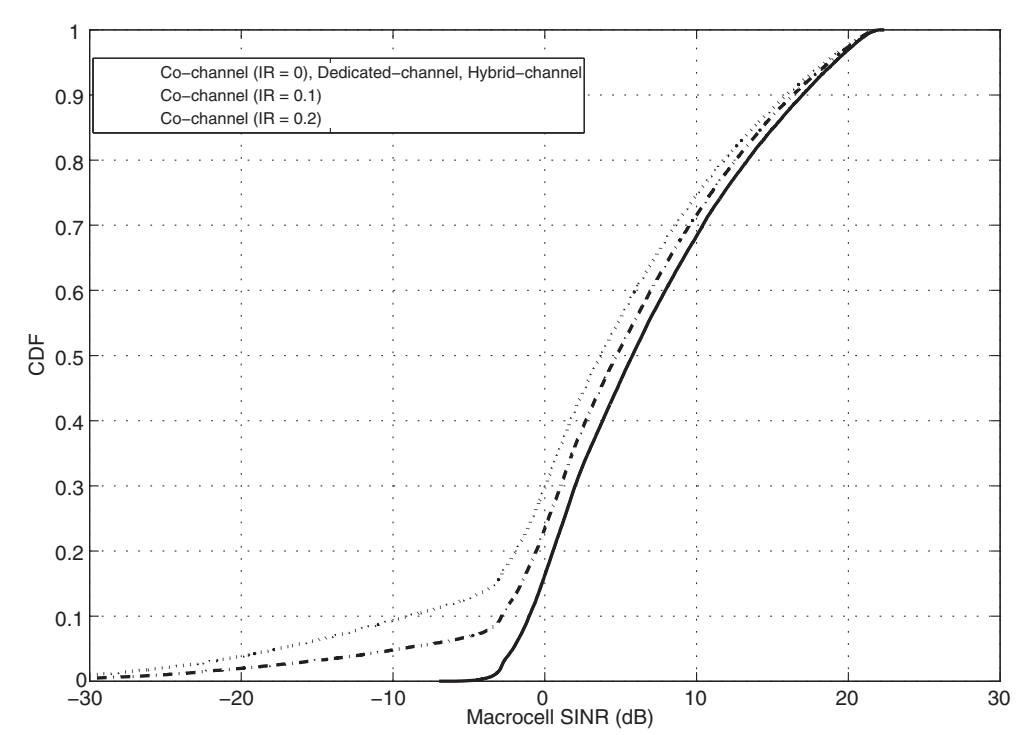

Figure 5 SINR of macrocell users vs. SSR $\rho$. 


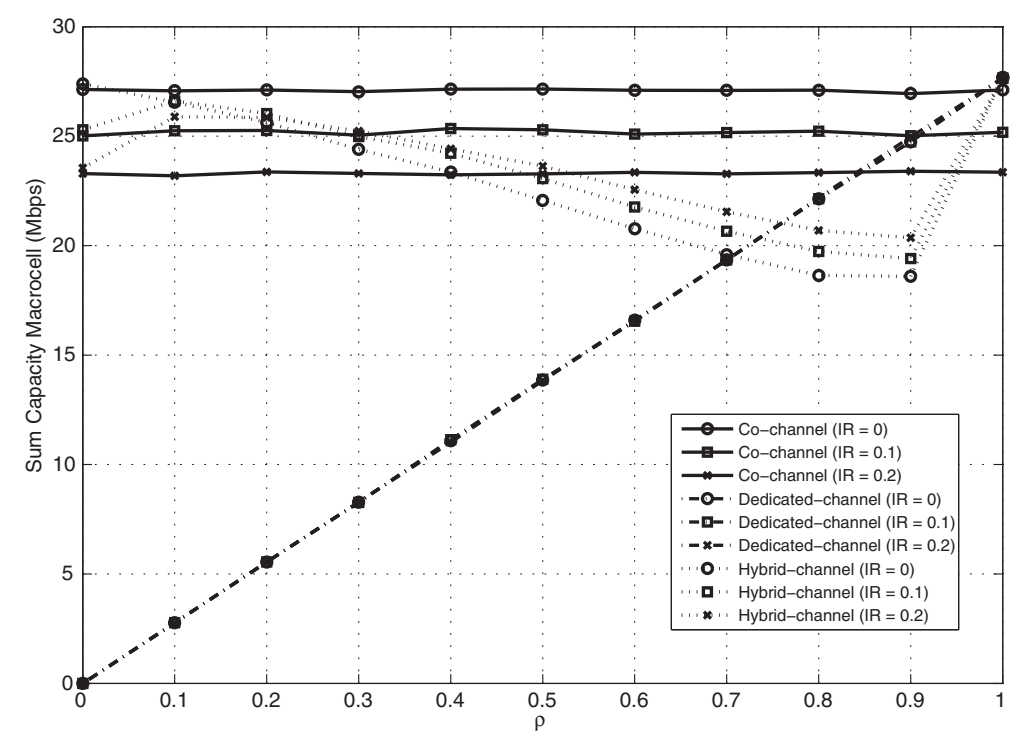

Figure 6 Sum capacity of macrocell vs. SSR $\rho$.

$\rho=0$ and dedicated channel at $\rho=1$. Co-channel macrocell sum capacity decreases with increasing IR, and dedicated channel capacity does not change with IR since femtocells do not interfere with macrocells. Note that hybrid approach does not let sum capacity of macrocell decrease below a level in Figure 6 and protects the victim users which are under severe interference. For $\mathrm{IR}=0.1$ and $\mathrm{IR}=0.2$, the hybrid approach sum capacity of macrocell first increases with $\rho$ comparing to co-channel. By scheduling the victim users in dedicated portion of the spectrum in hybrid approach, the maxmin scheduler achieves the highest sum capacity around $\rho \simeq 0.2$. However, as $\rho$ further increases, this behavior changes and sum capacity of macrocell decreases since max-min scheduler assignees the users to the dedicated channel portion aggressively to increase the victim user (5-percentile) capacity.

In Figure 7, hybrid channel 5-percentile capacities are always better than both co-channel and dedicated channel. The 5-percentile capacities are maximized around $\rho \simeq 0.5$. We also investigate the femtocell sum capacities for various values and see that the similar behavior in Figure 4 is experienced therefore the figure is not given for the sake of brevity. IR does not affect the femtocell

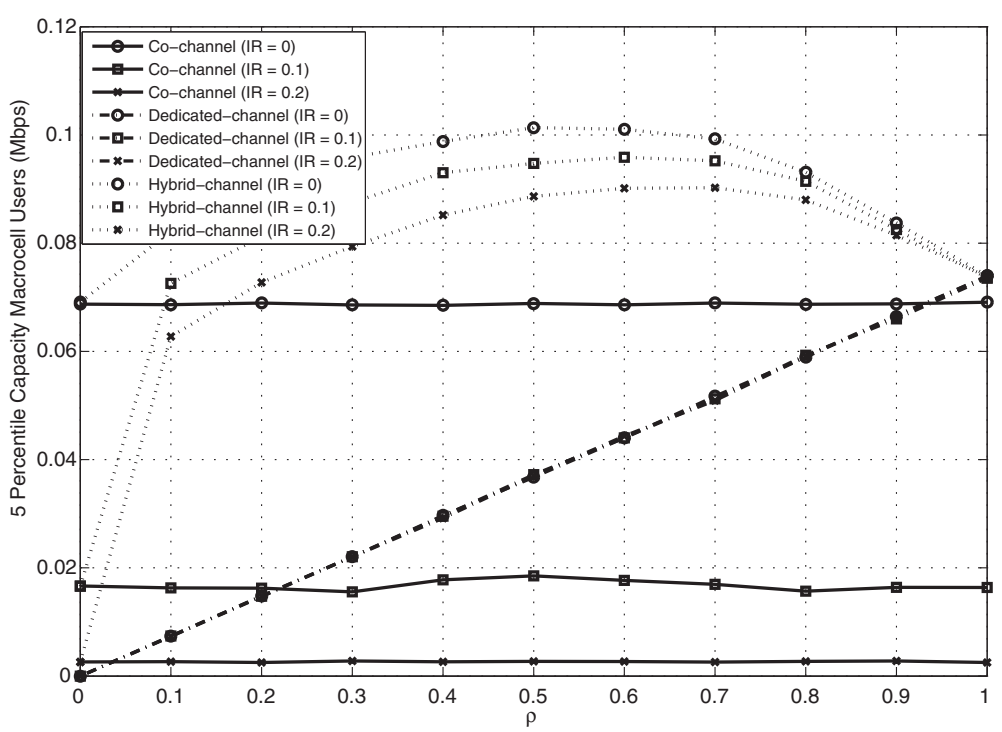

Figure 7 The 5-percentile capacity of macrocell vs. SSR $\rho$. 


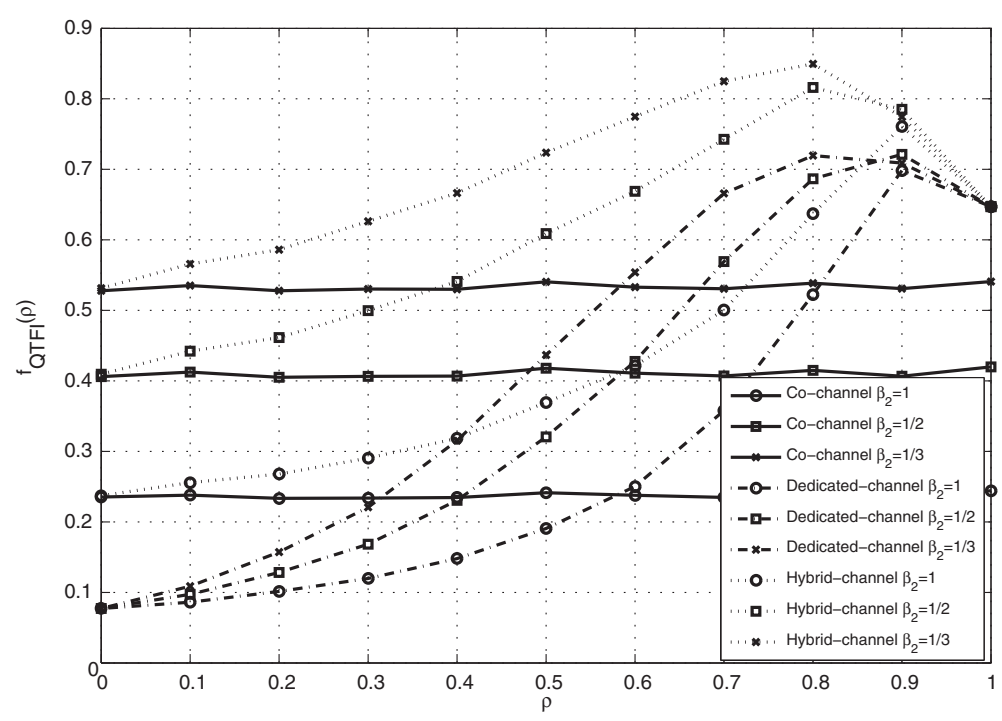

Figure 8 QoS-oriented fairness index $\left(f_{\mathrm{QTFI}}(\rho)\right)$ vs. SSR $\rho$ for various $\beta_{2}$.

capacities, and hybrid approach femtocell sum capacities decrease with increasing $\rho$. Figures 6 and 7 shows that for hybrid approach, selecting $\rho=[0.2,0.5]$ provides a good compromise between sum capacity and 5-percentile capacity of macrocell. On the other hand, smaller $\rho$ values maximize the femtocell capacity in hybrid approach (see Figure 4).

One way to analyze the distribution of macrocell and femtocell capacities is to use the fairness metric defined in Section 2. Figure 8 presents the fairness level of tiered network for co-channel, dedicated channel, and hybrid channel under various SSR and QoS parameters for IR = 0 . Note that hybrid approach $f_{\mathrm{QTFI}}(\rho)$ again converges to co-channel case at $\rho=0$ and dedicated channel at $\rho=1$. In co-channel scenario, femtocell user capacities are always larger than macrocell users, and therefore their fairness metric may only be moderately improved by changing $\beta$ in Figure 8 . For example in co-channel approach, for $\beta_{2}=1 / 3$, the expected femtocell user capacity is three times more than that of macrocell user; however, the $f_{\mathrm{QTFI}}(\rho)$ is still as low as 0.5 . On the other hand, hybrid channel approaches fairness metric is always above the co-channel and dedicated channel approaches. Note that for hybrid channel approach, the fairness maximization can be done for $\rho=0.8$, where it corresponds to one CC usage for femtocells out of five CCs in LTE-A [22].

To conclude, partitioning of available resources with the hybrid approach yields the best trade-off from capacity maximization, fairness, and QoS perspectives. The macrocell sum capacity maximization is done at $\rho \simeq$ 0.2 , macrocell 5 -percentile capacity maximization is done around $\rho \simeq 0.5$, and fairness maximization is done around $\rho \simeq 0.8$. Therefore, a network provider can able to operate the network depending on these three parameters according to the requirements.

\section{Conclusions}

In this paper, using HPPs, we study the sum capacities of co-channel, dedicated channel, and hybrid spectrum allocation methods for two-tier macrocell-femtocell networks For dedicated channel and hybrid approaches, optimum partitioning of the available spectrum resources between the macrocell and femtocell networks is derived analytically and analyzed for various scenarios. The results show that without using fairness criteria, the capacity maximizing allocation is done by allocating the whole spectrum to femtocells due to their spectrum reuse capability. Since this approach leads to a very unfair spectrum allocation, we propose a QoS-oriented fairness metric. By using this metric as a constraint for the spectrum allocation, we present a capacity maximizing spectrum allocation method which guarantees a specific level of fairness and QoS. From a network provider's point of view, partitioning of available resources with the hybrid approach yields the best trade-off from capacity maximization, fairness, and QoS perspectives. The findings in this paper may also be easily extended to time-domain resource coordination among macrocells and femtocells as specified in 3GPP Release-10 [22], where the duty cycle of blank subframes [23] may be optimized while jointly considering capacity maximization, fairness, and QoS constraints. 


\section{Endnotes}

${ }^{a}$ While the present paper will be mostly focusing on femtocell networks coexisting with a macrocell network, the proposed framework can be easily extended when other heterogeneous network entities are present in the system.

b Throughout the chapter, the terms shared spectrum and split spectrum will be used interchangeably with co-channel and dedicated channel, respectively.

c Note that the number of users in each cell is assumed to be known.

d It is important to note that this study considers a uniform distribution of BSs and MSs. Clusterization and hotspot deployment scenarios may yield different results [24]. This issue is not considered and left as future work.

e The analysis in this study can be applied to general models; however, the expressions are more complex, therefore we restrict ourselves for this case for the sake of brevity.

${ }^{f}$ Although (13) provides the SIR distribution, we can assume that the network is interference limited (SINR $\simeq$ SIR)

$\mathrm{g}$ As it is described in Section 3.3 and 3.4 for co-channel scenario $M=2$, HPP1 is mBS locations, HPP2 is fBS locations, $\left(v_{1}, v_{2}\right)=\left(\lambda, \lambda^{\prime}\right),\left(\mu_{1}, \mu_{2}\right)=\left(P, P^{\prime}\right),\left(m_{1}, m_{2}\right)=$ $\left(\lambda \pi r_{\min , \mathrm{m}}^{2}, \lambda^{\prime} \pi r_{\min , \mathrm{f}}^{2}\right), \Theta_{2}=\frac{\lambda^{\prime}}{\lambda}\left(\frac{P^{\prime}}{P}\right)^{2 / \delta}$, and $\left(d_{\max , 1}, d_{\min , 1}\right)=$ $\left(r_{\text {max }, \mathrm{m}}, r_{\text {min,m }}\right)$; for dedicated channel scenario $M=1$, HPP1 is mBS locations, $v_{1}=\lambda, \mu_{1}=P, m_{1}=\lambda \pi r_{\text {min, }}^{2}$, and $\left(d_{\max , 1}, d_{\min , 1}\right)=\left(r_{\max , \mathrm{m}}, r_{\min , \mathrm{m}}\right)$.

\section{Competing interests}

The authors declare that they have no competing interests.

\section{Acknowledgements}

The authors would like to thank Hazar Aki from University of South Florida for fruitful discussions and to Fujio Watanabe from DOCOMO Innovations Inc. for his support.

\section{Author details}

${ }^{1}$ Department of Electrical Engineering, University of South Florida, Tampa, FL 33620, USA. ${ }^{2}$ DOCOMO Innovations Inc., 3240 Hillview Avenue, Palo Alto, CA 94304, USA. ${ }^{3}$ Electrical and Computer Engineering, Florida International University, Miami, FL 33174, USA.

Received: 18 July 2012 Accepted: 2 April 2013

Published: 4 May 2013

\section{References}

1. Real Wireless Ltd, Strategies for mobile network capacity expansion. White Paper (West Sussex UK, 2010)

2. Qualcomm Inc, A comparison of LTE advanced HetNets and Wi-Fi. White Paper (2011). http://www.qualcomm.com/media/documents/ qualcomm-research-comparison-Ite-advanced-hetnets-and-wifi

3. R Madan, J Borran, A Sampath, N Bhushan, A Khandekar, T Ji, Cell association and interference coordination in heterogeneous LTE-A cellular networks. IEEE J. Select. Areas Commun. (JSAC). 28(9), 1479-1489 (2010)

4. V Chandrasekhar, JG Andrews, A Gatherer, Femtocell networks: a survey. IEEE Commun. Mag. 46(9), 59-67 (2008)

5. Nokia Siemens Networks, Macro+HeNB performance with escape carrier. 3GPP Standard Contribution (R1-101453), (Nokia, Beijing, 2010)
6. Z Bharucha, A Saul, G Auer, H Haas, Dynamic resource partitioning for downlink femto-to-macro-cell interference avoidance. EURASIP J. Wireless Commun. Netw. 2010(4), 1-12 (2010)

7. HA Mahmoud, I Guvenc, in IEEE 20th International Symposium on Personal, Indoor and Mobile Radio Communications, 2009. A comparative study of different deployment modes for femtocell networks (Tokyo, 13-16 September 2009), pp. 1-5

8. D Lopez-Perez, A Valcarce, A Ladanyi, G de la Roche, J Zhang, Intracell handover for interference and handover mitigation in OFDMA two-tier macrocell-femtocell networks. EURASIP J. Wireless Commun. Netw. 2010(4), 1-15 (2010)

9. I Demirdogen, I Guvenc, H Arslan, in IEEE 21st International Symposium on Personal, Indoor and Mobile Radio Communications Workshops (PIMRC Workshops). A simulation study of performance trade-offs in open access femtocell networks (Istanbul, Turkey, 26-30 September 2010), pp. 1-5

10. A Barbieri, A Damnjanovic, T Ji, J Montojo, Y Wei, D Malladi, O Song, G Horn, LTE femtocells: system design and performance analysis. IEEE J. Select. Areas Commun. 30(3), 586-594 (2012)

11. HS Jo, P Xia, J Andrews, Open, closed and shared access femtocells in the downlink. EURASIP J. Wireless Commun. Netw. 2012(4), 1-12 (2012)

12. N Saquib, E Hossain, LB Le, DI Kim, Interference management in OFDMA femtocell networks: issues and approaches. IEEE Wireless Commun. 19(3), 86-95 (2012)

13. M Erturk, H Aki, Guvenc I Guvenc, H Arslan, in IEEE Global Telecommunications Conference (GLOBECOM 2010). Fair and QoS-oriented spectrum splitting in macrocell-femtocell networks (Miami, 6-10 December 2010), pp. 1-6

14. DCR Jain, W Hawe, A quantitative measure of fairness and discrimination for resource allocation in shared computer system. DEC Technical Report 301, Digital Equipment Corporation, Hudson (1984)

15. A Kumar, J Kleinberg, in Proclamation of 41st Annual Symposium on Foundation of Computer Science. Fairness measures for resource allocation (Redondo Beach, 12-14 November 2000), pp. 75-85

16. X Gao, T Nandagopal, V Bharghavan, in Proceedings in IEEE Global Telecommunication Conference (GLOBECOM), Volume 6. Achieving application level fairness through utility-based wireless fair scheduling (San Antonio, 25-29 November 2001), pp. 3257-3261

17. J Andrews, F Baccelli, R Ganti, A tractable approach to coverage and rate in cellular networks. IEEE Trans. Commun. 59(11), 3122-3134 (2011)

18. S Mukherjee, in Proclamation of IEEE International Conference on Communication Workshops (ICC). UE coverage in LTE macro network with mixed CSG and open access femto overlay (Kyoto, 5-9 June 2011), pp. 1-6

19. V Chandrasekhar, J Andrews, Spectrum allocation in tiered cellular networks. IEEE Trans. Commun. 57(10), 3059-3068 (2009)

20. I Guvenc, Capacity and fairness analysis of heterogeneous networks with range expansion and interference coordination. IEEE Commun. Lett. 15(10), 1084-1087 (2011)

21. 3GPP Organizational Partners, 3rd Generation Partnership Project; technical specification group radio access network; evolved universal terrestrial radio access (E-UTRA); further advancements for E-UTRA physical layer aspects (release 9). 3GPP TR 36.814 Release 9 V9.0.0 (2010-03) (2010). http://www.quintillion.co.jp/3GPP/Specs/36814-900.pdf

22. S Sesia, T Issam, B Matthew, LTE the UMTS Iong term evolution: from theory to practice, 2nd edition. (Wiley, Chichester, 2011)

23. Samsung Electronics, CSI measurement issue for macro-femto scenarios 3GPP Standard Contribution (R1-106051). (Jacksonville, FL, 2010). http:// www.3gpp.org/ftp/tsg_ran/wg1_rl1/TSGR1_63/Docs/

24. C Coletti, L Hu, N Huan, I Kovacs, B Vejlgaard, R Irmer, N Scully, in Proceedings of IEEE Vehicular Technology Conference (VTC). Heterogeneous deployment to meet traffic demand in a realistic LTE urban scenario (Pacifico Yokohama, Yokohama, 6-9 May 2012), pp. 1-5

doi:10.1186/1687-1499-2013-121

Cite this article as: Ertürk et al.: Fair and QoS-oriented resource management in heterogeneous networks. EURASIP Journal on Wireless Communica tions and Networking 2013 2013:121. 